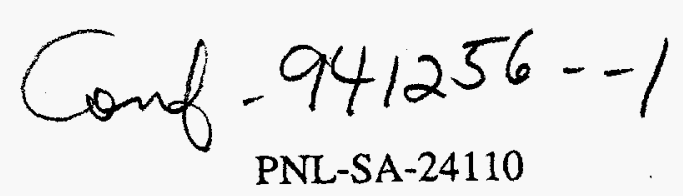

\title{
PREDICTING ENVIRONMENTAL RESTORATION ACTIVITIES THROUGH STATIC SIMULATION
}

\section{DISCLAIMER}

\author{
T. L. Ross \\ D. A. King \\ M. L. Wilkins \\ M. F. Forward
}

December 1994

\begin{abstract}
This report was prepared as an account of work sponsored by an agency of the United States Government. Neither the United States Government nor any agency thereof, nor any of their employees, makes any warranty, express or implied, or assumes any legal liability or responsibility for the accuracy, completeness, or usefulness of any information, apparatus, product, or process disclosed, or represents that its use would not infringe privately owned rights. Reference herein to any specific commercial product, process, or service by trade name, trademark, manufacturer, or otherwise does not necessarily constitute or imply its endorsement, recommendation, or favoring by the United States Government or any agency thereof. The views and opinions of authors expressed herein do not necessarily state or reflect those of the United States Government or any agency thereof.
\end{abstract}

Presented at the 1994 Winter Simulation Conference

December 11-14, 1994

Orlando, Florida

Prepared for

the U.S. Department of Energy

under Contract DE-AC06-76RLO 1830

Pacific Northwest Laboratory

Richland, Washington 99352 


\section{DISCLAIMER}

Portions of this document may be illegible in electronic image products. Images are produced from the best available original document. 


\title{
Predicting Environmental Restoration Activities Through Static Simulation
}

\author{
Terry L. Ross, Research Engineer \\ Dale A. King, Research Engineer \\ Mark L. Wilkins, Senior Engineer \\ Mary F. Forward, Research Engineer \\ Pacific Northwest Laboratory, ${ }^{(2)}$ Richland, WA 99352
}

Key Words: Environmental, Government

\begin{abstract}
This paper discusses a static simulation model that predicts several performance measures of environmental restoration activities over different remedial strategies. Basic model operation consists of manipulating and processing waste streams via selecting and applying remedial technologies according to the strategy. Performance measure prediction is possible for contaminated soil, solid waste, surface water, groundwater, storage tank, and facility sites. Simulations are performed for the U.S. Department of Energy in support of its Programmatic Environmental Impact Statement.
\end{abstract}

\subsection{Introduction}

The U.S. Department of Energy (DOE), including its predecessors, has had many missions over the years inciuding research and development in nuclear weapons, nuclear reactors, and energy development and production. These activities led to construction of facilities, production and testing of materials and hardware, and decommissioning of facilities. The execution of these activities created hazardous and radioactively contaminated areas and wastes.

A major mission of DOE today is to perform environmental restoration (ER) and waste management (WM) activiries on its complex. For this paper, ER involves activities relating to the renovation

(a) Pacific Northwest Laboratory is operated for the U.S. Department of Energy by Battelle Memorial Institute under Contract DE-AC06-76RIO 1830. 
and renewal of contaminated facilities, soil, groundwater, landfills, as well as other contaminated media. Waste management is concemed with managing and final disposition of stored and resulting wastes from ER activities.

To guide DOE in its ER and WM missions, a Programmatic Environmental Impact Statement (PEIS) is being developed. This PEIS will provide guidance for determining the most appropriate remedial action and WM philosophy for DOE to follow for all of its waste sites in all media. Specifically, this paper focuses on the ER portion of the PEIS. Management of secondary waste from ER is provided under a separate WM scope of the PEIS.

Successful completion of the PEIS requires a thorough understanding of the waste sites covered by the PEIS. This understanding requires analyzing the waste site before and after proposed remediation. The PEIS is evaluating seven proposed remediation strategies (called alternatives), of which five require unique analyses. These five are called engineering scenarios or cases (see Section 2.3). One alternative will eventually be chosen as policy to guide DOE's ER efforts.

Typical waste site analyses include prediction of appropriate remedial technologies, a determination of their effectiveness, identification of secondary waste streams generated, and estimation of the remedial action's performance parameters. For the PEIS, these parameters are public risk, worker risk, transportation risk, cost, labor requirements, and quantification of the amount of secondary waste.

To facilitate the analysis of DOE's complex, the ER PEIS project team decided to develop a model to aid in waste site analyses. The resulting model simulates environmental restoration under the five engineered scenarios. This paper describes this model.

\subsection{The Requirement For a Model}

Many factors led to the decision for developing a predictive environmental restoration system model. One reason for automating the analysis was because of the potential number of waste site analyses possible for the PEIS. Knowledgeable individuals associated with DOE estimated the number of contaminated waste sites to be in the order of several thousand.

Another reason was the number of the required calculations. The nature of the computations is not mathematically complex, but the number of logic steps as well as mathematical calculations involved for anaiyzing each waste site under each engineered scenario is extensive. A related reason for automated processing is to keep the detailed assumptions consistent between waste sites and cases.

The analysis team also anticipated numerous analysis iterations for the PEIS. As with any large multi-vendor project, many model runs are made while assumptions and analysis details are being refined. This project was no different; many analysis iterations were made. The lack of a model for iteration flexibility would have severely hampered the analysis effort. 
The above considerations led to the development a software model to aid in the analysis of the DOE complex. Due to the nature of the problem being solved, the model was not required to be a dynamic simulation. Extension of the current static simulation into a discrete-event simulation is discussed in Section 7.0.

\subsection{The Problem Domain}

rescarch and production fueclitivs

The DOE complex is a collection of DOE-owned and resenves, and located across the continental United States. For the purposes of the PEIS, only those sites hazardous or radioactive wastes are of concern. In general, these sites are located $\hat{h}$ installations where weapons-related research and production occurred (see Figure 1.1).

Because of fundamental differences in the remediation approach for various media, each contaminated site is classified as one of six types:

- Contaminated Facilities. Facilities generally refer to buildings or structures that require occupation or access by humans on a regular or semi-regular basis.

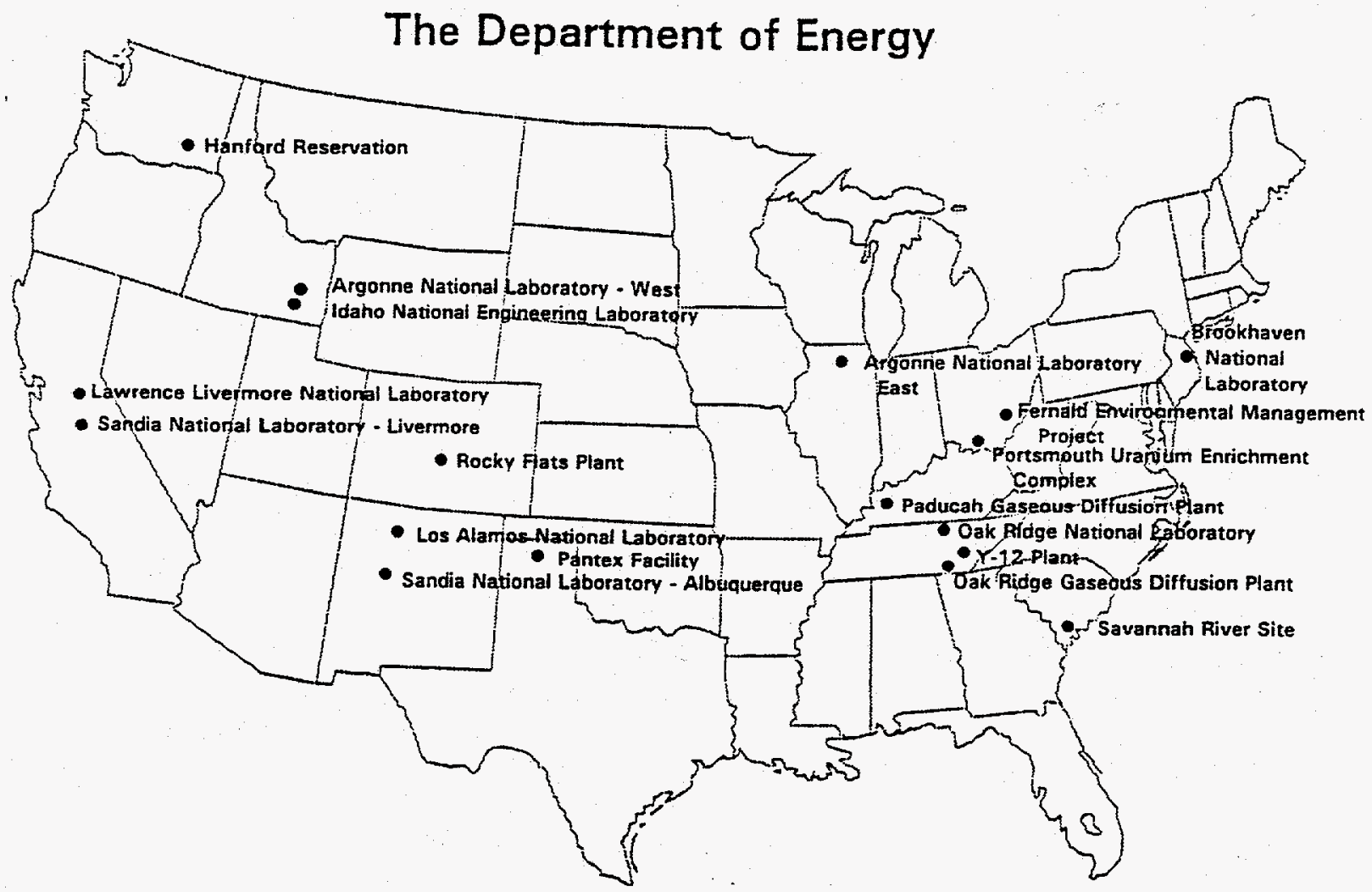

Figure 1.1. Major DOE Installations that are the Focus of Environmental Restoration Activities 
- Contaminated Groundwater Plumes. Contaminated groundwater sites can be either contained, uncontained, or perched aquifers.

- Liquid Containment Structures. This category includes structures used to temporarily store contaminated liquid effluents for later retrieval and treatment, or petroleum products for later retrieval and use. Structures used to transport contaminated liquid effluents or petroleum products from one location to another are also classified as liquid containment structures.

- Contaminated Soil Sites. Soil sites can consist of contaminated soils in the vadose zone or in the partially saturated zone.

- Solid Waste Sites. Solid waste sites are primarily used for the dumping or disposal of solid wastes or contained liquid wastes. Sites may or may not contain containerized (drummed) waste.

- Contaminated Surface Water sites. Contaminated surface water (standing or flowing) and sediments are included in this category.

These media types allow convenient categorization of waste sites at each installation.

\subsection{The Model}

The simulation model described in this section is named the Automated Remedial Assessment Methodology (ARAM). It is used to predict ER activities for five competing scenarios, across many waste sites and installations. The term prediction means to estimate ER activities "close enough" to allow for the selection of a case that will bias complex-wide restoration policy.

ARAM analyzes many waste sites from many installations, for a particular media, during one processing session. A complex-wide simulation consists of processing sessions for all media types for all cases.

\section{ANACYSS}

ARAM performs anolyzes on waste streams. Processing begins by assigning the waste site to the initial stream. As remedial action is simulated, new streams are generated from effluents of remedial technologies chosen during simulation. A waste site is not fully simulated until all waste streams for a given site are processed.

\subsection{Model Requirements}

A major requirement is flexibility. New waste sites need to be added to the analysis on demand. In addition, it is necessary for new technologies and associated performance measures to be easily 
added, changed, and updated. Finally, the overall treatment logic, which defines the conditions under which specific technologies are chosen, needs to be flexible.

Another model system requirement is the type of computer for running the model. Currently, the model requires a 486 IBM PC or compatible running Windows 3.1, with $4 \mathrm{MB}$ of RAM, and approximately $150 \mathrm{MB}$ of hard disk space available for a run using all the data for analysis. Since the model is typically $1 / O$ bound, it helps to have the hard disk connected to a local bus or to a PCI bus for added throughput.

For this application, ARAM run time is not critical. A 24-hour run time was specified as acceptable for a simulation of the complex. The model currently runs 1200 waste sites for approximately five scenarios, in roughly eight hours on three computers simultaneously.

A relatively quick analysis cycle is required because the analysis is not completed by simply running the model. Experts and engineers must manipulate the output data for verification, validation, summarization, extrapolation, and reporting. A faster analysis cycle is produced currently by managing model output with a database system. The database management system used is Microsoft Access ${ }^{\mathrm{th}}$ Version 1.1. The typical output database in Access measures $120 \mathrm{MB}$ for a model run of the complex.

A final requirement for the model development task involves allowing analysts to add new or change existing scenarios without recompiling the model. This has been achieved by developing the scenario logic in a flow charting package that produces files in a form ARAM can read. The feature is based on previously coded objects (or modules) that accept standard input and write standard output. These modules are reusable and form basic building blocks for remedial action.

\subsection{Available Waste Site Data}

Remedial analyses require detailed data for each waste site. Prior to the PEIS, little data existed that were suitable for waste site anaiyses for a majority of DOE's waste sites. The PEIS researchers performed a data gathering effort to collect as much data as possible for known waste sites.

ARAM is designed to run with minimal input data. Necessary input data falls into two major categories: waste site-specific data and contaminant-related data. Site data include such items as the media type, volume or payload of the site, linear dimensions or site area, and the environmental setting identifier. The necessary constituent-related data describe each of the contaminants by name, initial inventory, and/or concentration.

ARAM also accepts other input data and uses it depending upon the alternative and waste site processed. For example, the depth to groundwater may be required to determine if a specific technology is applicable. Therefore, the depth to groundwater is passed, even though it may not be used. If there are no data for depth to groundwater and the data are needed, ARAM will default to a conservative estimate. 
Risk factors are also supplied as input to ARAM. They provide a way for ARAM to estimate public risk based on a contaminant's concentration. Risk factors were developed by Oak Ridge National Laboratory for use in the PEIS analysis. These factors are developed for a particular installation, media, environmental setting, and contaminant.

\subsection{Solution Alternatives}

ARAM was designed to model different remedial altematives. Seven remedial alternatives were defined by DOE Headquarters; however, two of the seven alternatives had duplicate remedial analysis strategies. Therefore, five scenarios were required for complete analysis. Each of the five scenarios is defined below:

- Regulatory Driven (Unrestricted Access). This alternative is driven by onsite risk factors or concentration limits specified by regulations (whichever is most restrictive) to set the target level for remediation. The goal of the altemative is to reduce all contaminant concentrations to below regulatory limits and target concentrations as determined by the contaminant-specific onsite unit risk factors. In addition, institutional control is assumed to be lost, eliminating containment options that require long-term maintenance (caps, barriers, well-head controls, etc.). Further, these technologies do nothing to reduce contaminant concentration.

- Totally Restricted Land Use (i.e., DOE Retains Ownership). This alternative assumes that the entire installation (as currently defined) is restricted to public access, and only the risk to boundary receptors (e.g., people at the boundary of an installation) drives the remediation logic. In situ treatments and containment technologies are chosen first (if feasible) to reduce risk, followed by removal and treatment if containment is infeasible. Containment options are preferred, as the incurred cost and risk are generally lower for these technologies. Practical volumetric/areal limits are set to define when removal is more reasonable than containment.

- Semi-Restricted Land Use. For this alternative, institutional control is not retained by DOE, but some civil authority restricts the use of groundwater at the release site. Restricted use of the site further eliminates the incidental soil ingestion and food crop pathways for onsite receptors (for example people within the installation boundary). Remediation activities address both onsite and offsite receptors (with the exception of groundwater ingestion by the onsite receptor). Further, remediation must not preclude future land uses. Remediation measures must not require long-term maintenance because it is assumed that no agency is responsible for maintenance. These constraints essentially eliminate the use of containment technologies, with the exception of hydraulic controls for groundwater. 
- Health Risk Driven. This alternative seeks to weigh the cost of achieving public risk reduction, with cost being measured in terms of worker risk. Because this cannot be adequately addressed in one case without some very extensive logic, three sub-alternatives are evaluated. The results of these sub-alternatives provide the basis for assessing the relative costs of remedial approaches.

- Regulatory Driven. A separate ARAM analysis is not necessary for this portion of this alternative.

- Containment. Only containment technologies are applied in this case.

- Combination of Treament and Containment. This combination logic applies in situ treatment first, followed by containment, with ex situ treatment being a last resort as required to meet concentration targets. "Hot spot" treatment of groundwater and soils is applied to reduce contaminant concentrations to levels where containment technologies reduce residual risk to below targets.

\subsection{Performance Parameters}

Analyzing ARAM output involves comparing the treatment alternatives defined previously based on various performance parameters. The key performance measures are site status, DOE WM waste loads, costs, labor, worker risk, transportation risk, public risk, and complex-wide estimation. These performance measures are described in the following paragraphs.

- Site Status. For each site evaluated under a given ER strategy, one of four possible outcomes (final site status) is predicted:

- Successful. The suggested combination of treatments is estimated to be successful in meeting the contaminant concentration targets defined by the treatment strategy, based on the initial contaminant concentrations and the applicability and effectiveness of each of treatments applied by ARAM.

- Unsuccessful. Although one (or more) treatment is identified as applicable to the waste site based on the available data, contaminant concentrations are estimated to remain above concentration thresholds even after the site is treated to the limits of the technologies within ARAM.

- No Action. Based on available data, no contaminants are present at the site at levels exceeding concentration targets prescribed by the treatment strategy. 
- No Feasible Treatment. Although contaminants exist at the site at levels exceeding targets, no technologies are available in ARAM that appear to remediate the site to below levels of concern.

- DOE WM Waste Loads. Many treatment methods generate additional, or secondary, waste streams as a result of their application. An example of this is ex situ gas phase removal for contaminated groundwater plumes. Volatile contaminants removed from the contaminated groundwater are transported in a contaminated air stream to secondary treatment. The air is then circulated through granular activated carbon (GAC). Contaminants adsorb to the GAC, which becomes a secondary waste stream.

The volume and types of secondary wastes generated by a particular treatment are an important performance parameter for comparing competing strategies. Since additional cost and risk are incurred by WM to treat the secondary waste streams, one goal of each treatment strategy is to minimize these volumes.

Each waste stream that is to be sent to WM is assigned a physical classification. This designator classifies the physical form of the waste stream. The physical classification is dependent upon the treatment technology that produces it, not upon contaminant content.

The chemical classification of each secondary waste stream is determined by the original contaminants of concern at the site. The waste stream is assigned a classification based on the contaminants of concern present in the secondary waste stream.

In addition to the physical and chemical classifications, each waste stream routed to WM is assigned a radioactivity classification. Unlike the determination of chemical classification, radioactivity classifications are assigned based on the presence of specific radionuclides in the secondary waste stream.

- Costs. Costs for each treatment method are estimated by multiplying unit cost factors by the volume (or area) being treated. For example, excavation costs are based on the volume of soil being excavated, while constructed barrier costs are calculated from the perimeter of the site being contained. Separate costs are estimated for construction (capital) and operation and maintenance (O\&M).

The estimated cost is also an important performance parameter for each treatment strategy, for obvious reasons. Costs estimated by ARAM are direct costs for technology application and do not include indirect costs such as permitting and institutional controls.

- Labor. Similar to the unit cost factors, unit labor factors are used to estimate the amount of construction and operating labor required to apply a given treatment technology. Labor estimates, like the cost estimates, are broken into capital and O\&M. 
- Worker Risk. ER worker risk depends on the specific treatment methods that are applied. Some ER technologies, such as extraction wells, pose less risk to the worker than technologies such as excavation. A lower worker risk means the technology is less risky to the humans that operate and maintain them than another technology.

- Transportation Risk. Another key factor in comparing the relative performances of the various treatment strategies is transportation risk. For each volume of material brought onsite to support ER treatments, and for each secondary waste stream that must be transported either to a permanent disposal facility onsite or a centralized facility offsite, an associated transportation risk is estimated. Similar to worker risk, one goal of the technology selection logic is to minimize transportation risk.

- Public Risk. For treatment strategies that are driven by risk reduction (e.g., the health riskdriven strategy), public risk becomes the primary driver of the treatment logic. Public risk can be reduced by removal and destruction of contaminant inventory or retardation of risk (along specified pathways). Some technologies remove contaminant inventory (e.g., excavation followed by ex situ contaminant separation), while others reduce the mobility of contaminants, reducing the public risk from air, groundwater, surface water, or direct exposure.

\subsection{Complex-Wide Estimation}

The performance measures computed by ARAM include only the sites where enough data have been gathered to be analyzed by the model. Estimation of the entire DOE complex is required by the project. Therefore, an extrapolation method has been developed by the analysts to estimate the total expected performance parameter by installation. The complex estimate is the sum of all the installation estimates.

The extrapolation method takes the performance parameters calculated from ARAM and adds the additional estimates for the sites not analyzed. The estimated performance parameter is derived from developing a cluster of sites (with the same performance characteristics), computing the average performance parameter for the cluster, then multiplying the average performance measure by the number sites not analyzed.

Flexibility is built into the method for developing clusters. The cluster can be defined by one or more sire types for one or more installations. The result is a flexible methodology that is defendable and allows for conservative estimates for the total amounts of the desired performance measure. 


\subsection{Input/Output}

The I/O section contains an input module that creates streams from the input database (currently $\mathrm{dBase} \mathrm{IV}$ ) and an output module that writes the output data to tab separated text files. The input files are read using CodeBase $5.0^{\text {th }}$.

The input module creates a "stream" from each site that is read from the database. Streams are the basic unit of data in PRISM. In the current version, they contain, among other things, the following pieces of information:

- dimensions (length, width, area, volume) of the contaminated area

- concentrations of contaminants

- remediation target concentrations

- classification of contaminants

- general site information.

The data are stored in a generic structure to allow easy modification for other types of models.

\subsection{User Interface}

The user interface, developed with Visual Basic $2.0^{\text {th }}$, provides the mechanism for specifying the parameters and input files for a running a single case. It aiso provides the capability to automatically run multiple cases without user interaction.

\subsection{Modules}

PRISM contains a large library of technology modules and decision modules. These modules are coded specifically for the ARAM model and have implicit knowledge about the data in the stream structures. To solve other types of problems, a new suite of modules could be developed. The two main types of modules are technology and decision modules. The technology modules contain the domain-specific knowledge. They perform various calculations on the streams and output one or more streams. The decision modules do not modify streams; they simply make a decision based on the stream data and pass the stream along one of two possible routes. End nodes are a third type of module that accept streams, but they do not output any streams. The end nodes perform various data reporting tasks. All of the modules were developed as $C$ functions with standard call interfaces using Borland" $\mathrm{C}++3.1$. 


\subsection{Processor}

The processor in PRISM uses a queuing and routing algorithm that is independent of the domainspecific knowledge contained in the streams. The components of the processor were developed as $\mathrm{C}++$ classes using Borland ${ }^{\oplus} \mathrm{C}++3.1$. The internal flow diagram objects are created from the charts produced by Chartist-2 ${ }^{\mathrm{TM}}$ from Novagraph.

\subsection{How the Model Processes Sites}

This section provides a general overview of the general algorithm used to process data through the flow diagrams. The algorithm overview is shown in Figure 4.1.

PRISM performs all processing on a site-by-site basis. It reads a site and all corresponding contaminant information from the various databases and creates a stream, which is then routed through the flow diagram.

The inputs and outputs to all modules are streams. If a module has three outputs, then it will produce up to three streams. An example module is shown in Figure 4.2. The contents of the output streams (volumes and concentrations) are determined by the module definition. In general, technology modules produce a stream for each defined output arc. Each module also has a "reject" stream (not shown in Figure 4.2) that specifies the destination of streams for which the particular module is not applicable. When the module does not accept a stream, it sends the input stream unchanged along the reject arc. If it does accept an input stream, it sends an output stream along each of the two other output arcs.

Decision modules always have two output arcs; a "yes" and a "no." An example of a decision module is shown in Figure 4.3. For each input stream, these produce a single output stream on one arc depending on the contents of the stream and the module definition.

Because modules can produce more than one stream, PRISM stores streams for processing in a first-in-first-out (FIFO) queue. This causes the diagrams to be processed in a breadth-first order. When there is more than one stream to process, PRISM chooses the first stream on the queue and sends it to the next appropriate module on the flow chart. This continues within a single chart for a single site until all streams have been deleted (through end nodes) or have been sent to sub-diagrams.

If necessary, the order of processing can be controlled through a custom module that puts a "holding" weight on streams in the queue. Streams with lower hold weights are processed first. 


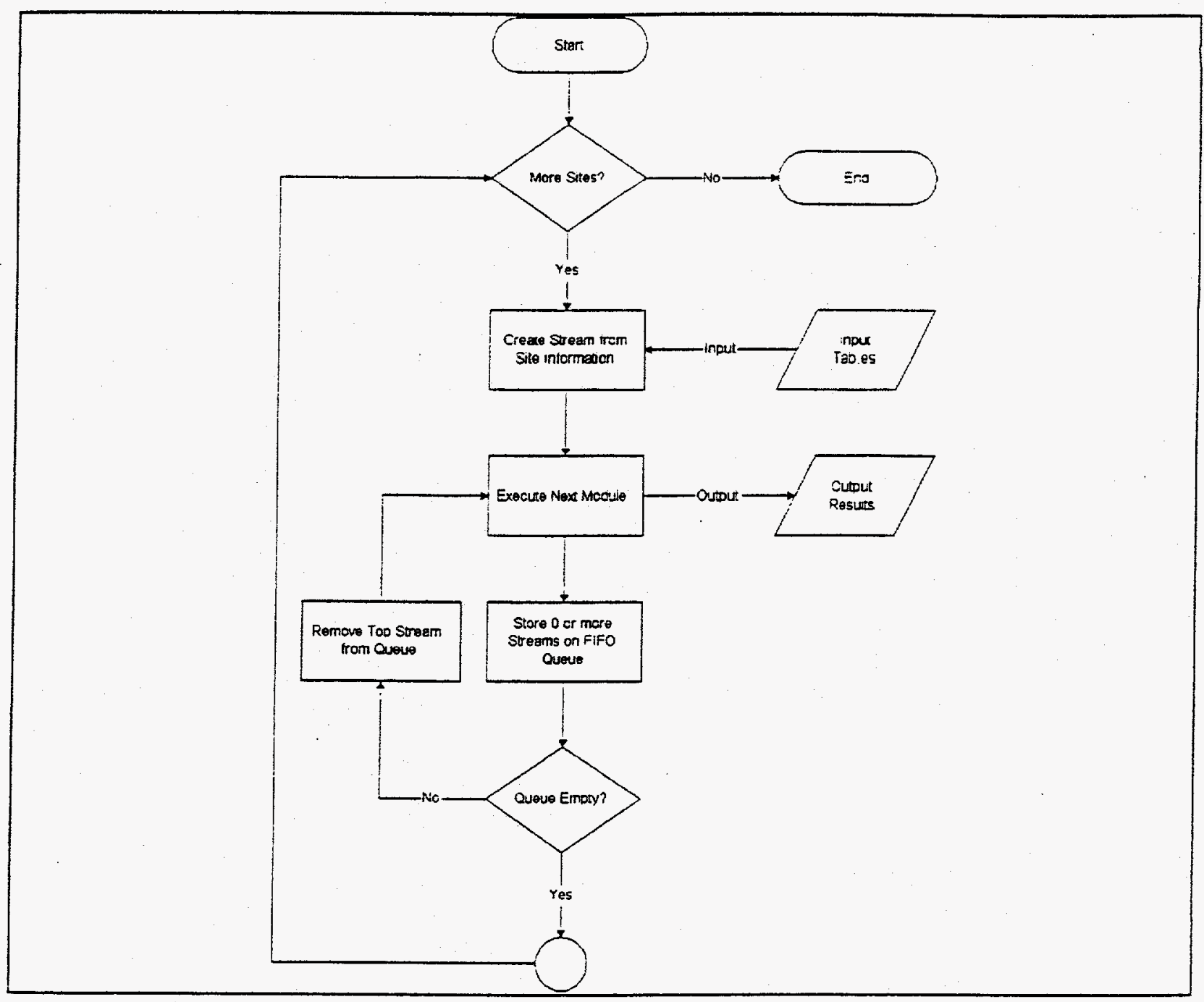

Figure 4.1. PRISM Flow

\subsection{Logic Diagrams for PEIS Alternatives}

For each media type, up to five separate logic diagrams exist that cover seven different PEIS land use alternatives (or cases), as previousiy described. For some media types, a single logic diagram serves for multiple alternatives. The logic diagrams are constructed using a standard logic flow approach and determine the subset of treatments that are applicable for each alternative, the necessary conditions for their application (in addition to the conditions specified in the method descriptions), the priority given to treatment methods, and the routing of secondary streams produced by each method. Although the logic diagrams are meant to give preference to certain treatment methods as determined by the ordering of the logic, the location of one method relative to another on the diagram does not imply any chronological ordering of the treatments. For example, the regulatory-driven groundwater 


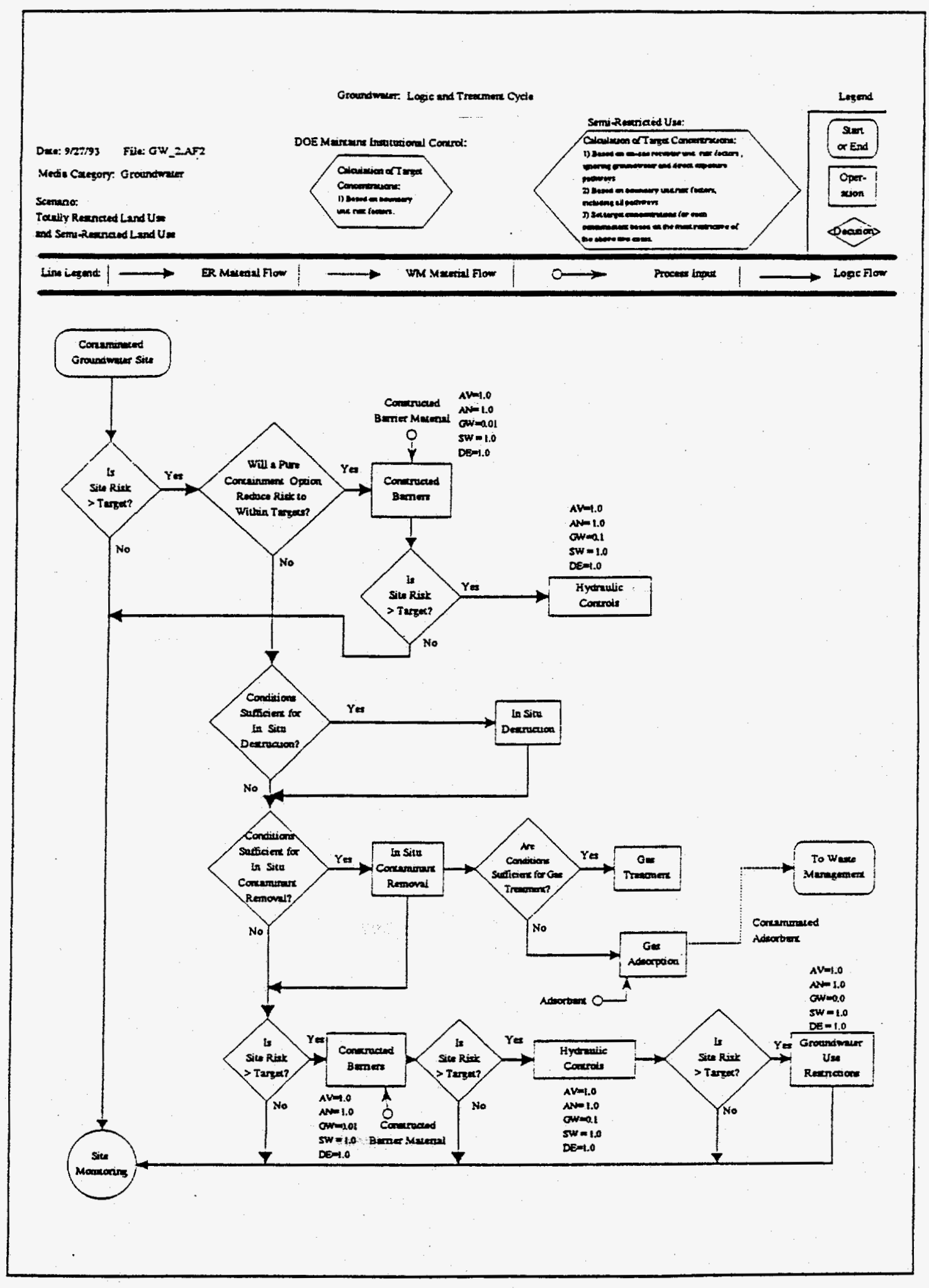

Figure 4.4. Example Logic Diagram 


\subsection{Material Balance Methodology}

One property of all technology modules is the material balance it performs on the streams it processes and creates. The material balance methodology between technology modules is virtually unique. Each individual material balance is developed using the following general approach:

- set basic conditions that must be satisfied for selection of the technology category

- define parameters used in determining applicability of a method

- establish rules for eliminating technology category from consideration when use would not be feasible

- apply material balance to contaminated medium (waste volume and contaminant concentration estimation)

- provide insight into basis for effectiveness and mass balance calculations for a technology category

- provide guidance for cost estimation for technology category based on the technologies represented by that category.

Overall assumptions made in developing the material balances are listed below:

- Parameters defined in the material balances correspond to data available as a result of PEIS data gathering activities.

- Contaminants are present in common chemical forms (oxides, hydroxides, and carbonates) in soils, groundwater, and surface water, and the contaminants are assigned to classes according to that form (e.g., when classifying a contaminant as soluble versus insoluble).

The algorithms for these material balances were derived primarily from technology descriptions developed for RAAS (White and Bryant 1993). Supplemental information for technology applicability to specific contaminants and technology effectiveness was drawn from other technical resources (PNL 1993 and EPA 1992).

\subsection{Verification and Validation}

Any model must be verified and validated before results can be trusted. Verification and validation of ARAM are discussed in detail below. 


\subsection{Verification}

Verification of ARAM had three distinct parts: unit testing, regression based testing, and typical output testing.

Unit testing is an approach used to test each technology and decision module for results anticipated by the methodology. Each module is given enough input states to verify all possible output states. As errors are found when comparing module output to the methodology, necessary changes are made and testing performed again. Unfortunately, unit testing is not enough to verify all the components of the model operate correctly as a system.

Over time, the ARAM code can became fairly complicated. Therefore, the software engineers implement regression based testing to ensure that incremental modifications to the model produce only the desired effects. Testing starts by comparing the results of the test run to verified results. If changes in output appear to be the result of the code change, the software engineers pass the executable model to the analysts and experts for verification. If not, the software engineers debug their changes until the model performs as desired.

Once the software engineers release an executable version or the code, the model is run and results are obtained. The results are summarized by installation, alternative, and media. Errors are found by comparing the summary data to expert opinion and previous model runs. Even if no errors are found this way, waste sites from each media are selected and tested. These exercises verify the required changes occur as desired.

\subsection{Validation}

Complete validation of this type of model is virtually impossible for two reasons. First, very little restoration of radioactive and mixed wastes (waste that contains both radioactive and hazard constituents) sites has been performed. This makes simulation of a remediated site for comparison purposes impossible. Second, the alternatives used to guide model execution have never been used to guide remediation. These two facts make complete validation unachievable.

The project has been able to pseudo-validate ARAM. This occurs by having the output of several model runs reviewed by experts for correct (expected) technology application, costs, labor requirements, risk reduction, and effluent volumes by waste type. The experts involved with validation have extensive experience with hazardous contaminated waste sites, understand bench-scale remediation and treatment of radioactive and mixed wastes, have studied current remediation literature, and participate in environmental technology development. The validity of the model is only as good as the experts who validated it. 


\subsection{Model Applicability To Other Problem Domains}

Although the methodology and model were developed for DOE, ARAM can be applied to other problems. In general, ARAM is useful for solving "what if" problems on one or more different scenarios. In addition, ARAM is useful for analyzing multiple waste sites from one or more different geographical locations.

ARAM would be a benefit to those industrial users that have several hazardous contaminated waste sites scattered in many different regions in the country and/or abroad. ARAM would compute planning-level numbers for analysts to determine the size of acomprehensive remediation project. These numbers could be used as a basis for environmental restoration ${ }^{\text {scemarios }}$

The U.S. Department of Defense (DOD) is similar to the DOE in that it controls several different geographical locations that need environmental restoration. Examples include the military bases that have petroleum, solvent, and other spills as well as facility decommissioning. ARAM would be well suited to analyze these type of restoration problems.

ARAM could help EPA plan for and manage the many waste sites under its control. The previously discussed studies are as well suited for EPA as for DOE.

Similar studies using ARAM could be performed by State and local agencies responsible for publically owned waste sites and oversight of privately owned waste sites. ARAM could also be used to conceptually verify the intent of those parties responsible for cleanup.

\subsection{Future Research and Development}

The development of ARAM has produced many opportunities for additional research and development. It is envisioned that the extension of the current static simulation into a dynamic simulation would allow the results of ARAM to be used to estimate the required technology capacities and utilizations over the life of ER activities. Capacity requirements are imperative for refining the capital cost portion of the ER plan. Utilization in conjunction with capacity levels dictate the cost of operational and maintenance support required for the ER.

A discrete event paradigm most likely would be adopted. This implementation would allow waste streams to be "events" pushed onto an event list. More attributes would need to be added to the stream to allow for the necessary record keeping. Finally, and event list and handler would need to be added to the basic ARAM methodology. 
Another future extension could be developing regulatory scenarios. These scenarios could predict the impacts of EPA regulation to sites just starting to plan for remediation. This would give ARAM a broader applicability for remedial analyses. In addition, it would enable governing agencies to simulate the impacts of new and/or changed laws and policies on the performance parameters generated by ARAM.

\subsection{References}

White, M. K. and J. L. Bryant. 1993. ReOpt ${ }^{\text {rx }}$ v2.1. PNL-7840, Rev. 1, Pacific Northwest Laboratory, Richland, Washington.

Pacific Northwest Laboratory (PNL). 1993. RAAS Prototype 2.5 - Remedial Action Assessment System-Remedial Investigation/Feasibility Study Document (DRAFT). PNL-8752, Pacific Northwest Laboratory, Richland, Washington.

U.S. Environmental Protection Agency (EPA). 1992. VISITT - Vendor Information System for Innovative Treatment Technologies, Version 1.0. EPA-542-r-92-001, EPA Office of Solid Waste and Emergency Response, Washington, D.C. 